\title{
EDITORIAL
}

\section{Lymphoedema screening: setting the standard}

Existing literature which is changing practice should be scrutinised, in the interest of all women at risk for lymphoedema after breast cancer (BC). Bundred et al.'s prospective, multicentre trial of 1100 women made several solid findings, and novel screening recommendations presented may assist in incorporating lymphoedema screening into standard of care.

British Journal of Cancer (2020) 123:1-2; https://doi.org/10.1038/s41416-020-0848-0

\section{MAIN}

Screening for lymphoedema after breast cancer (BC) surgery is recommended ${ }^{1-5}$ but not standard of care currently. There is no universal diagnostic criteria for BC-related lymphoedema (BCRL); criteria are often correlated but not interchangeable, ${ }^{6}$ hindering research progress. ${ }^{7}$ The recent trend towards screening for subclinical BCRL with bioimpedance spectroscopy (BIS) and treating at a lower threshold lacks evidence. Resources for BCRL screening and treatment are scarce and quality of life (QOL) is impacted by BCRL. Therefore, this movement should be scrutinised, and Bundred et al. ${ }^{8}$ in the current issue of the British Journal of Cancer have provided the needed data to make evidence-based recommendations with this study.

The authors conducted a multicentre prospective study of 1100 patients comparing multi-frequency BIS with arm volume measurement. The aims of the study included determining which test has better accuracy, identifying factors predicting $B C R L$, and understanding its effect on QOL. Patients were measured with perometry (arm volume) and BIS at baseline and throughout 5 -year follow-up. BCRL was defined as a relative arm volume increase $(\mathrm{RAVI}) \geq 10 \%$ or BIS L-Dex increase two or three standard deviations from baseline ( $>7.5$ or $>10$ respectively). Patients with $\mathrm{RAVI} \geq 10 \%$, swelling in the lower arm or hand, or BCRL symptoms were diagnosed with $B C R L$ and fitted for compression.

Of note, Bundred et al. ${ }^{8}$ found that $8.3 \%$ of patients had volume differences $\geq 200 \mathrm{~mL}$ between arms at baseline. This is consistent with Sun et al., ${ }^{9}$ where $2.9 \%$ and $28.3 \%$ of patients had arm volume differences at baseline of $10 \%$ and $5 \%$, respectively. This study highlights the importance of baseline measurements, echoing the finding that not incorporating baseline measures results in up to $50 \%$ misdiagnosis. ${ }^{9}$ Preoperative baseline measurement should be the standard. ${ }^{1-5}$

This study found a 2-year BCRL incidence of $22.4 \%$ via RAVI, and $45.2 \%$ and $57.6 \%$ via BIS (L-Dex increase $\geq 10$ and 7.5 , respectively). ${ }^{8}$ While they note that there is no gold standard definition of BCRL, they use perometry as benchmark in this study. In the established literature, 2-year incidence of BCRL in patients undergoing axillary clearance is not as high as $57.6 \%$ (via BIS in Bundred et al. ${ }^{8}$ ). A highly cited meta-analysis found that the 2-year incidence from 18 studies of patients with axillary dissection was $19.9 \%$ (range $13.5-28.2 \%$ ), ${ }^{10}$ consistent with the $22.4 \%$ incidence via $R A V I \geq 10 \%$ in this paper.

Studies have found that BIS is responsible for significant false positives, ${ }^{11}$ and in another study of 58 patients diagnosed with BCRL via ICG lymphography (the true gold standard), 21 had a normal L-Dex via BIS-a $36 \%$ false negative rate. ${ }^{12}$ Bundred et al. ${ }^{8}$ aptly point out that the literature base touting BIS's ability to detect subclinical BCRL is limited-studies lack baseline measurements and long-term follow-up to identify patients who return to a normal after an abnormal L-Dex reading without intervention. ${ }^{13}$ BIS is not established to identify subclinical BCRL, and from Bundred et al.'s study ${ }^{8}$ on 1100 patients, RAVI $\geq 10 \%$ is recommended for screening.

Although RAVI $5 \%$ to $<10 \%$ is a risk factor for progression to $\mathrm{RAVI} \geq 10 \%,{ }^{14}$ Bundred et $\mathrm{l}^{8}{ }^{4}$ correctly state that the threshold for early treatment with compression is not evidence based. Stout et al.'s observational study, ${ }^{15}$ which treated $\mathrm{RAVI} \geq 3 \%$ with a sleeve for 4 weeks, found arm volume reductions of $4.1 \%$, which were maintained over 4.8 months. This was a small study $(n=43)$ without a control group; arm volume may have decreased without intervention. Further, Specht et al. found that only $18.7 \%$ of patients with an RVC of $5 \%$ to $<10 \%$ occurring $>3$ months after surgery progressed to RVC $\geq 10 \% .{ }^{14}$ More research is needed before start treating BCRL at RAVI $3 \%$ or $5 \%$.

We applaud Bundred et al.'s addition to the literature around risk factors and QOL. ${ }^{8}$ They had several interesting findings, for example that BMI at surgery predicted reduced $\mathrm{QOL}$ and progression of BCRL even after sleeve fitting. Improved education and resources are needed. They found that a high percentage of patients with BCRL report swelling or heaviness, which supports many national organisation's recommendation ${ }^{1-5}$ of incorporating symptoms into BCRL screening.

Bundred et al. ${ }^{8}$ found that after compression sleeve application, patient QOL scores increased for patients with RAVI $\geq 5 \%$ but not for those with $\mathrm{RAVI}<5 \%$. This is one of the first studies showing that patients may find solace in treating BCRL early. One other study found improvements in depression and anxiety following treatment for BCRL. ${ }^{16}$ This may result from increased patient sense of autonomy in management of a highly feared sequelae of $B C$ treatment.

Finally, Bundred et al. ${ }^{8}$ summarised screening recommendations based on their novel scoring system for BCRL progression risk: risk increased as scores increased. A small percentage (12\%) of patients with a low risk score at one month progressed to RAVI $\geq 10 \%$. In contrast, $76.7 \%$ of patients with a high-risk score progressed. Bundred et al. aptly observe that the risk of progression in the low risk group cannot be ignored, recommending allotment of resources towards high-risk populations whilst educating lower risk groups to ensure self-referral with any new symptoms. ${ }^{8}$ If resources allow, we recommend screening all patients; 65 patients in the low risk group in this study progressed to BCRL. 
In conclusion, we applaud Bundred et al.'s well-conducted and important study. ${ }^{8}$ This study was unable to demonstrate any evidence supporting BIS for detection of subclinical BCRL. Baseline and longitudinal arm volume measures and symptoms monitoring is imperative for screening. The effectiveness of early intervention with a sleeve at RAVI $5 \%$ to prevent progression to RAVI $\geq 10 \%$ has not been established. We add that BCRL screening is important to detect clinical BCRL in its early stages (RAVI $\geq 10 \%$ ), not just subclinical BCRL. Although recommended, it has unfortunately not been implemented as standard of care.

This trial has many strengths that need to be considered. In the interest of all women at risk for lymphoedema after BC, we must scrutinise the literature which is changing practice for its limitations. Future directions include controlled, long-term studies that delineate the ability of BIS to identify subclinical $B C R L$ and identify the true threshold for early treatment. The standard needs to be set, but it must be based on a strong research foundation.

\section{ACKNOWLEDGEMENTS}

We would like to acknowledge the contribution of Tessa Gillespie, Program Manager, MGH Lymphedema Research Program, for her editing of the paper.

\section{AUTHOR CONTRIBUTIONS}

Both authors have contributed equally to the paper in terms of concept, writing, editing and review of final draft.

\section{ADDITIONAL INFORMATION \\ Ethics approval and consent to participate Not applicable.}

Consent to publish Not applicable.

Data availability Not applicable (editorial)

Competing interests A.G.T. is on the Scientific Advisory Board of Puretech Health and is a previous consultant in VisionRT. A.G.T. has been loaned equipment from ImpediMed for use in investigator-initiated clinical trials. C.L.B. is on the Scientific Advisory Board of Puretech Health. These associations are unrelated to this paper.

Funding information The project was supported by Award Number R01CA139118 (A.G.T.) and Award Number P50CA08393 (A.G.T.) from the National Cancer Institute. The content is solely the responsibility of the authors and does not necessarily represent the official views of the National Cancer Institute or the National Institute of Health. This program is supported by the Adele McKinnon Research Fund for Breast Cancer-Related Lymphedema (A.G.T.) the Olayan-Xefos Family Fund for Breast Cancer Research (A.G.T.), and the Heinz Family Foundation (A.G.T.).

Note This work is published under the standard license to publish agreement. After 12 months the work will become freely available and the license terms will switch to a Creative Commons Attribution 4.0 International (CC BY 4.0).

Publisher's note Springer Nature remains neutral with regard to jurisdictional claims in published maps and institutional affiliations.
Cheryl L. Brunelle ${ }^{1}$ and Alphonse G. Taghian ${ }^{2}$

${ }^{1}$ Department of Physical and Occupational Therapy, Massachusetts General Hospital, Boston, MA 02114, USA and ${ }^{2}$ Department of Radiation Oncology, Massachusetts General Hospital, Boston, MA 02114, USA

Correspondence: Alphonse G. Taghian (ataghian@mgh.harvard.edu)

\section{REFERENCES}

1. McLaughlin, S. A., Staley, A. C., Vicini, F., Thiruchelvam, P., Hutchison, N. A., Mendez, J. et al. Considerations for clinicians in the diagnosis, prevention, and treatment of breast cancer-related lymphedema: Recommendations from a multidisciplinary expert ASBrS panel: Part 1: definitions, assessments, education, and future directions. Ann. Surg. Oncol. 24, 2818-2826 (2017).

2. International Society of Lymphology. The diagnosis and treatment of peripheral lymphedema: 2016 consensus document of the International Society of Lymphology. Lymphology 49, 170-184 (2016).

3. National Lymphedema Network Medical Advisory Committee. Position Statement of the National Lymphedema Network: Screening and Measurement for Early Detection of Breast Cancer-Related Lymphedema. (2011).

4. Stout, N. L., Binkley, J. M., Schmitz, K. H., Andrews, K., Hayes, S. C., Campbell, K. L. et al. A prospective surveillance model for rehabilitation for women with breast cancer. Cancer 118, 2191-2200 (2012).

5. Denlinger, C. S., Sanft, T., Baker, K. S., Broderick, G., Demark-Wahnefried, W., Friedman, D. L. et al. Survivorship, Version 2.2018, NCCN Clinical Practice Guidelines in Oncology. J. Natl Compr. Cancer Netw. 16, 1216-1247 (2018).

6. Armer, J. M. \& Stewart, B. R. A comparison of four diagnostic criteria for lymphedema in a post-breast cancer population. Lymphat Res. Biol. 3, 208-217 (2005).

7. O'Toole, J., Jammallo, L. S., Miller, C. L., Skolny, M. N., Specht, M. C. \& Taghian, A. G. Screening for breast cancer-related lymphedema: the need for standardization. Oncologist 18, 350-352 (2013).

8. Bundred, N. J., Foden, P., Todd, C., Morris, J., Watterson, D., Purushotham, A. et al. Increases in arm volume predict lymphoedema and quality of life deficits after axillary surgery: a prospective cohort study. British Journal of Cancer https://doi. org/10.1038/s41416-020-0844-4 (2020).

9. Sun, F., Skolny, M. N., Swaroop, M. N., Rawal, B., Catalano, P. J., Brunelle, C. L. et al. The need for preoperative baseline arm measurement to accurately quantify breast cancer-related lymphedema. Breast Cancer Res. Treat. 157, 229-240 (2016).

10. DiSipio, T., Rye, S., Newman, B. \& Hayes, S. Incidence of unilateral arm lymphoedema after breast cancer: a systematic review and meta-analysis. Lancet Oncol. 14, 500-515 (2013).

11. Barrio, A. V., Eaton, A. \& Frazier, T. G. A prospective validation Study of bioimpedance with volume displacement in early-stage breast cancer patients at risk for lymphedema. Ann. Surg. Oncol. 22, 370-375 (2015).

12. Qin, E. S., Bowen, M. J. \& Chen, W. Diagnostic accuracy of bioimpedance spectroscopy in patients with lymphedema: a retrospective cohort analysis. J. Plast. Reconstr. Aesthetic Surg. 71, 1041-1050 (2018).

13. Seward, C., Skolny, M., Brunelle, C., Asdourian, M., Salama, L. \& Taghian, A. G. A comprehensive review of bioimpedance spectroscopy as a diagnostic tool for the detection and measurement of breast cancer-related lymphedema. J. Surg. Oncol. 114, 537-542 (2016).

14. Specht, M. C., Miller, C. L., Russell, T. A., Horick, N., Skolny, M. N., O'Toole, J. A. et al. Defining a threshold for intervention in breast cancer-related lymphedema: What level of arm volume increase predicts progression? Breast Cancer Res. Treat. 140, 485-494 (2013).

15. Stout Gergich, N. L., Pfalzer, L. A., McGarvey, C., Springer, B., Gerber, L. H. \& Soballe, P. Preoperative assessment enables the early diagnosis and successful treatment of lymphedema. Cancer 112, 2809-2819 (2008).

16. Can, A. G., Eksioglu, E. \& Cakci, F. A. Early detection and treatment of subclinical lymphedema in patients with breast cancer. Lymphat Res. Biol. 17, 368-373 (2019). 